\title{
7. Placebo and Nocebo Phenomena
}

The Latin word 'placebo' means 'I shall please', and the opposite term 'nocebo' means 'I shall harm'. In the twelfth century a placebo was an evening service made in order to please a deceased person. In the fourteenth century the content of the term had changed a bit. It came to refer to the simulated tears shed by a professional mourner in connection with deaths and memorial services. The term appears in medical literature in the 1780s, and it then meant that a doctor was more ready to please and to follow the wishes of the patient than to be truly medically useful. In 1906, the prominent American physician, Richard Cabot (1868-1939) stated that placebo giving was nothing but quackery.

\subsection{What is the placebo problem?}

The modern expression 'the placebo effect' evolved during the 1950s, when Randomized Controlled Trials (RCT) became more commonly used. It means that a patient's symptoms or a disease disappears merely because the patient expects that they will disappear as an effect of a certain biomedical treatment. From a purely conceptual point of view, this biomedical placebo effect had better be placed in a wider conceptual framework, where it is merely one of several different kinds of placebo effects, and where, in turn, placebo effects are merely one of several conceptually possible kinds of psychosomatic curing.

All placebo effects are constituted by self-fulfilling expectations; the patient is cured simply because he believes and expects to become cured by some treatment. In order for a placebo effect to arise, the applied treatment must, for the patient, have the meaning or symbolic significance that it will cure him. The patient shall consciously and sincerely (but falsely) believe that he is being given a real treatment of some sort for his somatic problems. The existence of placebo effects can be investigated in relation to all kinds of health treatments that are claimed to be effective. Here is a list of four different kinds of possible placebo effects: 
- the placebo effect in relation to biomedical treatments

- the placebo effect in relation to psychotherapeutic treatments

- the placebo effect in relation to healing (see Figure 1)

- the placebo effect in relation to self-healing (e.g., meditation regarded as a spiritual activity, and made in the belief that through this activity one will be cured).

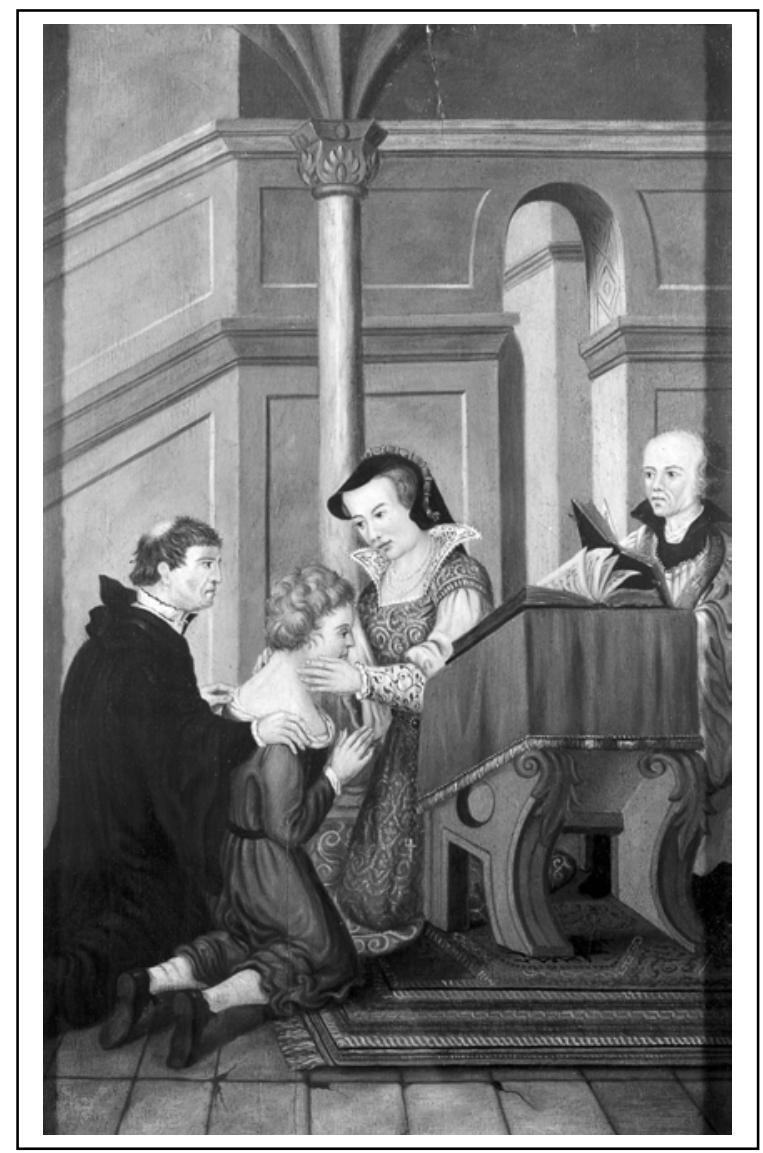

Figure 1: "A man with a skin disease came to him and begged him on his knees, 'If you are willing, you can make me clean.' Filled with compassion, Jesus reached out his hand and touched the man. 'I am willing' he said, 'Be clean!' Immediately the leprosy left him and he was cured."(Mark 1:40-42, Matthew 8:2-3, Luke 5:12-13) It is not only Jesus who is believed to have cured patients in this way. For more than 700 years the appointed kings and queens of France and England were regarded as possessing supernatural abilities. Just like Jesus the king had only to touch the patient for him to be cured. In the present painting Queen Mary (1516-1558) provides the 'Royal touch' to a patient, probably in the year 1553. 
All the four listed kinds of effects are by definition psychosomatic effects, since expectations are psychic (mental) entities. But they are not the only psychosomatic effects that might be in need of more careful philosophical-scientific investigations. Behind claims such as 'his strong will saved his life', there is a belief that a psychic will-to-live can be a causal factor in curing an otherwise deadly disease. Behind sayings such as 'his promotion/falling-in-love/winning-the-prize made his problems disappear', there is a belief that some somatic illnesses and diseases can become better only because the patient for some accidental reason enters a more positive psychological mood. Therefore, let us distinguish between at least the following three kinds of possible psychosomatic curing or (to cover a broader range) health improvement:

- psychosomatic health improvement due to expectations that one will be better

- psychosomatic health improvement due to a will to become better

- psychosomatic health improvement due to positive psychological moods.

We will mainly present the problem of psychosomatic curing as it appears in relation to the biomedical placebo effect that occurs in RCTs. To study the placebo effect in relation to healings and psychotherapies has problems of its own; for instance, the placebo treatments must be construed in another way. However, from general theoretical considerations, we find the following two theses plausible:

(a) if the biomedical placebo effect exists, then this is indirect evidence for the existence of placebo effects in psychotherapies and healings

(b) if there is psychosomatic health improvement by means of selffulfilling expectations, then this is indirect evidence for the existence of psychosomatic health improvement through will power and psychological moods.

When the persons in the control group of a normal RCT are given dummy pills or some other kind of fake treatment, this is not done in order to study and elucidate the placebo effect. On the contrary, the intention is 
to use what happens in the control group as a means to isolate the biomedical effect in the experimental group. Mostly, the control group of an RCT is simply called 'the control group', but sometimes when the RCT is placebo controlled the group is also called 'the placebo group'. In our discussion below, it is important that the label 'control group' is chosen. Why? Because the outcome in this group might be due also to other factors than the literal placebo effect. Let us explain by means of our earlier introduced informal formula $\mathrm{B}=\mathrm{T}-\mathrm{N}$; or $\mathrm{T}=\mathrm{B}+\mathrm{N}$, meaning that the Total effect in the experimental group contains both the real Biomedical effect of the treatment as well as an effect that is similar to the Nontreatment that obtains in the control group. However, the last effect has to be regarded as, in turn, containing three different possible factors:

(1) a literal placebo effect (P)

(2) an effect caused by spontaneous bodily curing (S)

(3) an 'effect' that is merely a statistical artifact (A).

That is, we can informally write: $\mathrm{N}=\mathrm{P}+\mathrm{S}+\mathrm{A}$.

When one is only interested in testing whether there is a biomedical effect, one can rest content with the variable $\mathrm{N}$ and look only at $\mathrm{B}=\mathrm{T}-\mathrm{N}$, but if one is interested in the placebo effect as such, then one has to discuss all the factors that may contribute to the value of $\mathrm{N}$. The general question is whether or not $\mathrm{P}$ should always be ascribed the value zero. If it should not, then many specific questions regarding the placebo effect arise. Then, of course, it ought to be investigated when, why, and how biomedical placebo effects occur; how their strength can vary; and what different kinds of biomedical placebo effects there are.

In a rather recent meta-analysis (Hrobjartsson and Gotzsche 2001), it has been claimed that (some minor pain reductions apart) the effects of clinical placebo treatments do not differ from mere non-treatment. To put their and many others - view bluntly, the placebo effect is a myth. Apart from the biomedical effect there is, these researchers claim, only spontaneous curing (in the patients) and unnoticed (by some researchers) statistical artifacts. That is, $\mathrm{N}=\mathrm{S}+\mathrm{A}$, since $\mathrm{P}$ is always zero; and $\mathrm{B}=\mathrm{T}-(\mathrm{S}+\mathrm{A})$. Good researchers try to take $A$ into account before they talk about the real effect; some not so good researchers sometimes forget it. 
The spontaneous course of a disease is the course the disease will follow if no treatment is provided, and no other kind of intervention is made. What in this sense is spontaneous may differ between different kinds of individuals. Therefore, one had better talk about the spontaneous courses of a disease. Be this as it may. To investigate the spontaneous course as such has its own problems, and we will not touch upon these. For the philosophical purposes now at hand, it is enough to have made the variable S clearly visible. So, what about the variable A?

When telescopes and microscopes were first used in science, optical illusions created by the instruments were quite a problem (see Chapter 2). It was hard to say whether some seen phenomena were caused by the instrument or whether they were real features of the investigated objects. Analogously, when statistics was first used in empirical science, statistical illusions created by the mathematical machinery appeared. It was hard to say whether some data were mere statistical side effects or representations of real empirical phenomena. Famous is the story about how the English polymath and statistician Francis Galton (1822-1911) first thought that he empirically had found a causal relationship in a statistical material; called its statistical measure 'coefficient of reversion'; realized that it was in fact a result of his mathematical-statistical presuppositions; and then re-named into 'coefficient of regression'. He was reflecting on an inheritance experiment with seeds, where he compared the size of the 'parents' with the size of their offspring. The kind of fallacious reasoning he discovered is nowadays called 'the regression fallacy'.

In pure mathematical statistics, there is a theorem called 'regression towards the mean' that is concerned with a comparison between a pregiven primary sample and a secondary sample. Both samples belong to the same population, which has a given probability distribution on a certain variable. If the mean value $\left(\mathrm{X}_{1}\right)$ of this variable in the primary sample is far away from the mean value $(\mu)$ of the whole population, then the mean value of the randomly chosen secondary sample $\left(\mathrm{X}_{2}\right)$ will probably be closer to $\mu$ than $X_{1}$ is. 'Going' from the primary sample to the secondary sample means 'going' from $X_{1}$ to $X_{2}$, and thereby 'regressing' towards $\mu$. When the two samples represent empirical data, the pure mathematical probability statements have been exchanged for some kind of objective probability statements (Chapter 4.7). The purely mathematical regress 
towards the mean may then in the empirical material falsely be taken as an indication that there is some kind of cause that makes it the case that $\mathrm{X}_{1}$ in the primary sample turns into $\mathrm{X}_{2}$ in the secondary one.

When the regression fallacy is presented in this verbal fashion, it may seem odd that any modern researcher should ever take a regress towards the mean to represent causality, but in statistical investigations with many dimensions the regression can be hard to discover. Consequently, the converse mistake - to find only a mathematical regression to the mean where there in fact is a causal relation - is also quite possible. In our opinion, much discussion around statistical artifacts seems to be permeated with the problems of the interpretation of probability statements that we highlighted in Chapter 4.7. There are also reasons to think that the kind of discussion of the causal relation that we presented in Chapter 6.2 might have repercussions on how to view presumed regression fallacies.

We regard the general problem of the existence of a biomedical placebo effect as not yet finally solved. That is, this book does not try to answer questions such as 'Is there anywhere a biomedical placebo effect?' and 'Can there be some kind of psychosomatic curing?' We try to make these issues clearer in the hope that such a feat might improve future medicophilosophical reflections and medico-scientifical investigations. We will, though, in the next section write in the traditional RCT-way as if there were a biomedical placebo effect. If there is none, then some of the things we say have in some way to be re-interpreted as being comments on spontaneous curing.

On the assumption that there is a biomedical placebo effect, some people have been classified as being high placebo responders and others as being low responders. A couple of recent investigations by means of position emission tomography (Petrovic et al 2002, Lieberman et al 2003, Zubieta et al 2005) and functional magnetic resonance scan (Eisenberger et al 2003, Petrovic et al 2005) lay claims to show that there is a specific area in the brain that can be associated with the placebo and the nocebo effects. In this area there is a significant difference in brain activity between high and low placebo responders (see Figure 2). 


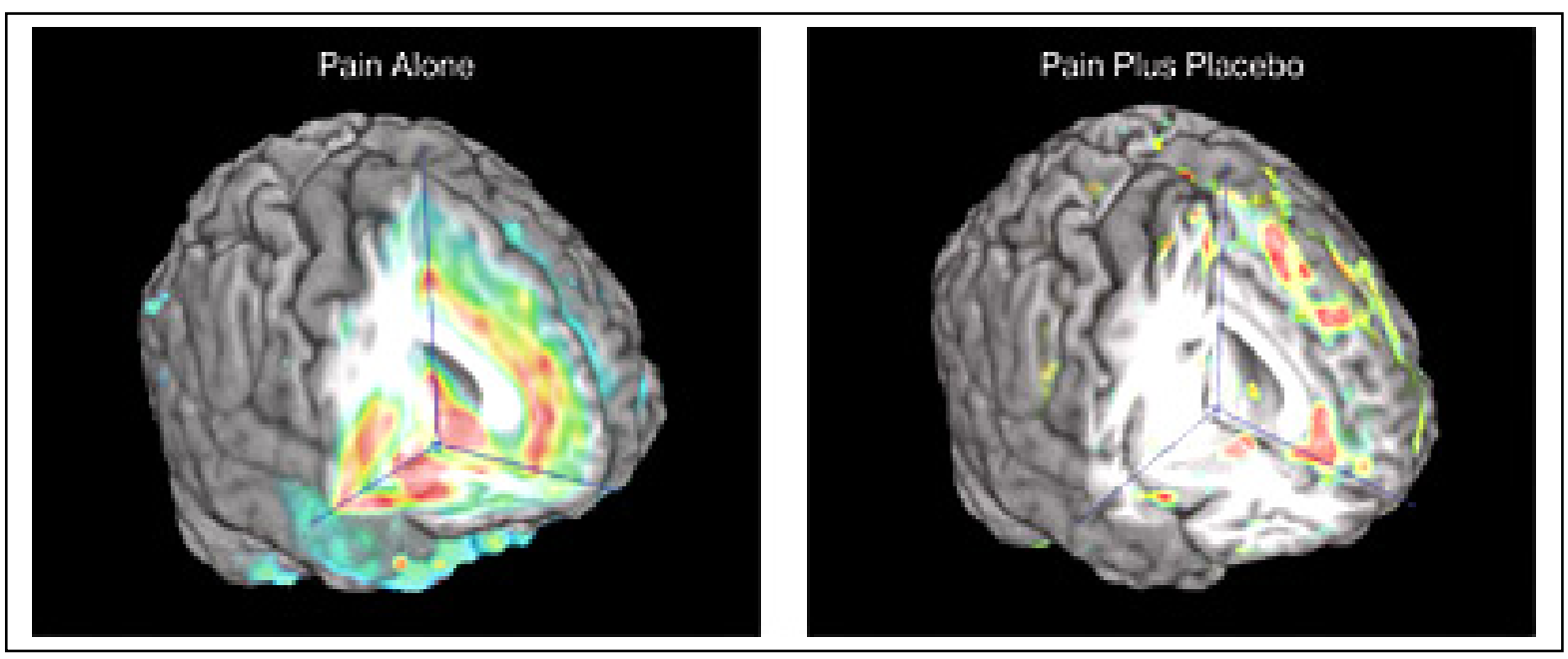

Figure 2: The pictures above are so-called molecular PET (positron emission tomography) images. They show the activity of the endogenous opioid system on $\mu$-opioid receptors in humans in sustained pain without (to the left) and with (to the right) the administration of a placebo. The difference is much more visible in color pictures. (Credit: Jon-Kar Zubieta.)

\subsection{Variables behind the placebo effect}

There are studies around the biomedical placebo effect as such. It is by no means a constant effect, and it varies with many variables. In some RCTs the placebo effect is close to zero and in others close to 100 percent. Some relevant variables - which may mutually interact - are: a) characteristics of the patients; b) characteristics of the doctors/providers; c) the seriousness of the diseases; d) the nature of the medical treatments; and e) the context and setting of the treatments. In a sense, all the factors (b) to (e) have to do with how the individual patient perceives the corresponding factors.

(a) The patient. In relation to the biomedical placebo effect, there seems to be two broad categories of patients: 'high placebo responders' and 'low placebo responders'. However, there has been a tendency to over-interpret these labels and make the corresponding features too basic properties of the persons in question. How an individual patient reacts depends on the cultural setting; how the patient feels; how ill the patient is etc. In one setting a patient may be a high responder and in another a low one. 
Nonetheless, there is a difference. Moerman has shown that high responders improve more than low responders in both the experimental group and the placebo group. In one investigation the mortality rate (after five years) among those who took three real tablets three times per day (of a cholesterol-lowering treatment) against myocardial infarction was $15 \%$ for high responders and $24.6 \%$ for low responders. Among those who received dummy tablets the mortality rate was $15.1 \%$ and $28.2 \%$, respectively. That is, in this study there was no difference between the experimental and the placebo groups with respect to the outcome, but there was such a difference between the group of high responders and the group of low responders.

(b) The doctor. What the patient-provider relationship looks like affects the degree of the biomedical placebo effect. Confidence in the skill and positive attitude of the doctor makes the placebo effect higher. However, what actually creates confidence and trust in the patient may also vary. An authoritarian attitude might create high confidence in some patients and low in others; and the same goes for a non-authoritarian attitude. Listening to the patient, capacity of being emphatic, respecting the patient's autonomy, etc., contribute to high placebo effect and vice versa. A study (Olsson et al 1989) shows that patients suffering from tonsillitis, who were treated both medically correctly and with empathy (including giving explanations of various sorts to the patients) were cured significantly more promptly compared to patient who were treated only medically correctly.

(c) The nature of the disease. In general, it seems to be the case that the more pain a disease is associated with, the higher is the placebo effect. For instance, in angina pectoris the placebo effect is higher during winter than in summertime when the conditions causing angina pectoris are more significant. On the other hand, some studies show remarkably uniform values. In a compilation of 22 RCTs concerning the treatment of pain with different drugs (weakest, aspirin; medium, codeine; and strongest, morphine), the placebo effect was on average within the 54-56 \% interval in all trials. Diseases where the placebo effect has been shown to be rather high include angina pectoris, peptic ulcer, claudicatio intermitens, and allergic conditions; but even some types of cancer have been reported to 
show a non-negligible placebo effect. One might well speculate about whether the prestige of certain diseases (both in view of the patient and in view of the doctor) might influence the magnitude of the placebo effect, but this has not yet been studied. Some diseases such as acute myocardial infarct and endocrine dysfunctions have been estimated by doctors and medical students to be more prestigious than others such as schizophrenia and fibro-myalgia (Album 1991).

(d) The nature and meaning of the treatment. Dummy injections seem to be more effective than dummy pills; and when administrating dummy pills or capsules, the color, size, taste and form of the pills are of importance. The number of pills seems also to have impact on the placebo effect. This has been shown in a meta-analysis of 71 RCTs for the treatment of peptic ulcer; four placebos per days is significantly more effective than two. Capsules (of chlordiazepoxide) seem to be more placebo effective against anxiety than pills - ceteris paribus; but here even cultural aspect may influence the effect. As stated by Moerman, Italian females associate the blue color with the dress of the Virgin Mary, who is seen as a protective figure and thus blue pills or capsules might be used as sedatives or sleeping pills. In contrast to this, Italian men associate the blue color with the national Italian Soccer team, the Azzurrii, which means success, strength, and excitement. Blue sleeping pills might accordingly be effective to Italian females and work less well for Italian men. Moerman notes in passing that Viagra is marketed in a blue pill. Sham operations have also proven to give rise to a certain placebo effect, e.g., sham chest surgery (Cobb et al. 1959), sham operations (masteoidectomies) behind the ear for a disease (called Mb. Menniére) in the inner ear (Thomsen et al. 1983), and arthroscopic sham operations in osteoarthritis in the knee (Moseley et al 2002).

(e) The situation and the cultural setting. Treatments provided in a highly specialized and famous university hospital may give rise to a stronger placebo effect than an ordinary country side treatment by a general practitioner. Even the surrounding - a room with a view (compared to looking at a brick wall) - seems to influence recovery after gall bladder surgery. It seems also to minimize complaints to the nurse as well as need 
of painkillers and even scores for post-surgical complications. In a metaanalysis of 117 trials concerning the treatment of peptic ulcer with acid inhibitors, there was a large variation between the different countries involved. For instance, the trials from Brazil had a placebo effect that was significantly lower than in other countries; seven percent versus thirty-six. Part of this difference might be due to the fact that the Helicobacter pylori bacteria might be more common in Brazil compared to the rest of the world, but it does not explain why (six) studies from Germany (part of the same meta-analysis) show that the average of the placebo effect was $59 \%$. In Germany's neighboring countries, Denmark and the Netherlands, which in most respects are quite similar to Germany, the average placebo effect was $22 \%$ in the above mentioned six trials. The placebo effect is, however, not always high in a German context; in comparative placebo controlled trials concerning hypertension, the German studies showed the least improvement in the placebo treatment group. The natural culture as well as the setting and the nature of the treatment seem to influence the placebo effect.

If there is a biomedical placebo effect, then many factors that work in concert influence it. If there is no such placebo effect, then the conclusion is that there are many factors that influence what is called the spontaneous curing or natural course of an illness and disease. That is, there are no absolutely spontaneous or natural processes.

\subsection{Specific and unspecific treatment}

Many medical treatments are said to be unspecific. That is, one does not know exactly what is to be regarded as, so to speak, the real treatment in the treatment. That is, one has not (yet) been able to isolate or point out the specific substance supposedly functioning. The notion of specific treatment emerged in early biochemistry (1880s) when the function of enzymes was described in terms of a key-lock model. The model implies that to a certain lock there is only one key. A treatment is regarded as specific when we are able to point out the functioning mechanism (on a biomechanical or molecular level) of the treatment. There has also been talk about specific causes and effects in relation to other mechanism theories and disease processes. Specificity, etiology and pathogenesis have been central concepts in medicine. Etiology refers mainly to causes that are responsible 
for bringing about or initiating a disease; pathogenesis is concerned with the disease process. Intrusions of micro-organisms as causes of infections are examples of etiology, but the immunological reaction and consequences of the manifestation of the infection are examples of pathogenesis. The clinical medical paradigm has said that - ideally medical treatments should consist in specific treatment of specific diseases.

We have made this brief remark here in order to stress that placebo treatment and its effects are usually classified as being unspecific. Although it has been possible to understand some aspects of the placebo effect in terms of modern neurophysiology (neuro-peptides like endorphins) as well as in terms of Pavlovian conditioning, the effect is in general unspecific.

Research in modern biogenetics (including genomics and proteomics) is constantly developing, and if these efforts are rewarded, then we might in the future have pharmaceutical treatments which are specific not only in relation to general etiology and/or pathogenesis, but specific also in relation to the individual patient. Genetic knowledge about the patient's enzyme system, pharmacological kinematics, immunological system, weight, and so forth, might be of importance when choosing the right medicine and the right dosage. Probably, it will be more appropriate to call such treatments 'individuated' or 'tailored treatments' than call them 'specific treatments'.

\subsection{The nocebo effect and psychosomatic diseases}

In Section 1, we distinguished between three kinds of psychosomatic curing and four kinds of placebo effects. All these conceptual possibilities have a negative counterpart:

- psychosomatic health impairment due to expectations that one will become worse

- psychosomatic health impairment due to a will to become worse

- psychosomatic health impairment due to negative psychological moods. 
The first of these conceptual slots represents nocebo effects. That there is a nocebo effect means that there is a somatically healthy person that becomes somatically ill or worse merely because he expects to become ill or worse. Nocebo effects are constituted by negative self-fulfilling expectations. The second possibility relates to self-destructive persons as well as to persons that want to become ill or sick in order to benefit from this negative state of affairs in some other and life-quality increasing respect.

The third possibility represents that possibility that there might be psychosomatic illnesses and diseases. Traditional but nowadays debated examples are asthma, some allergies, and migraines. To folk wisdom belongs the view that one can become sick by sorrow and lost love. A somewhat spectacular example is the report of two hundred cases of socalled psychogenetic blindness among Cambodian refugee women who were forced to witness the torture and slaughter of their family members, a procedure conducted by the Khmer Rouge. The women themselves say that they have seen the unbearable and cried until they could not see, and it has not been possible to identify any organic changes or defects in their visual system (Harrington 2002). We will return to and discuss psychosomatic diseases in more detail in Section 7 below.

The nocebo effect comes in at least the following varieties:

(i) the nocebo effect in relation to biomedical conditions and treatments

(ii) the nocebo effect in relation to psychotherapeutic treatments

(iii) the nocebo effect in relation to anti-healing

(iv) the nocebo effect in relation to self-punishment.

(i) Here are some possible examples of the biomedical nocebo effect. It has been reported (Jarvinen 1955) that when the chief of a cardiac intensive unit conducted the rounds, the number of those who had another myocardial infarction doubled compared to ward rounds where the assistant doctor was in charge. Among geriatricians, the expression 'a peripety' is sometimes used to describe sudden unexpected deaths by elderly patients who receive a cancer diagnosis. The expression is derived from the Greek word 'peripeteia', which means a sudden reversal of 
something to its opposite. In many ancient plays such a change of scenery takes place when an unexpected messenger appears. Transferred to the geriatric context it means that elderly cancer patients, who according to the medical prognosis might continue to live for a year or more, die quickly only because they think that there is a somatic cause that will make them die quickly. This phenomenon has also been described in connection with Nazi concentration camps. If a watchman told a new prisoner that he should not expect to leave the place alive, the latter sometimes isolated totally, wandered around with an empty gaze, and eventually died.

(ii) The existence of self-fulfilling expectations that one will become worse from psychotherapy, can be discussed in relation to people who feel forced to enter such a therapy.

(iii) What we call 'the nocebo effect in relation to anti-healing' is what is traditionally called 'voodoo'. It has been claimed that persons can become sick merely because some other but special person tell them that he will, by means of his spiritual powers, make them sick. The most extreme example is the so-called 'voodoo death'. This is a phenomenon reported by anthropologists to exist in communities where the bonds to particular norms, values, and taboos are very strong (Cannon 1942). The medicine man in such cultures is both a judge and executioner, though not executioner in the traditional sense, he simply pronounces the sentence and then psychological mechanisms in 'the sinner' makes the rest. The phenomenon has been claimed to exist even in modern societies. According to Harrington (2002), a mother who one and the same day became aware of the fact that her son was both homosexual and suffering from AIDS, reacted by making a prayer in which she expressed the wish that her son should die because of the shame he had caused her. The son heard the prayer, and one hour later he died. The physician was surprised because the patient was not terminally ill.

(iv) Self-destructive people who believe in voodoo can of course try to curse themselves with some somatic disease. This would be an attempt at what we call 'the nocebo effect in relation to self-punishment'.

We will return to psychosomatic diseases, but not to the other conceptually possible psychosomatic phenomena mentioned in this brief taxonomic section. 


\subsection{The ghost in the machine}

The question whether psychosomatic health improvements and health impairments really exist touches a core issue in both the biomedical paradigm itself and in its relation to the sub-paradigm that we have labeled the clinical medical paradigm. There is a tension between the general paradigm and the sub-paradigm that we think deserves more attention than it has received so far.

The expression that constitutes the title of this section comes from the English philosopher Gilbert Ryle (1900-1976), who used it in his famous book, The Concept of Mind (1949). He maintains that the view that there is a mind, a psyche, or a mental substance is a ghost created by our language. If he is right, there can be no placebo and nocebo effects since there is no mind that can produce any somatic effects.

We have previously claimed that, with respect to the medical realm, the ontology of the biomedical paradigm is an epiphenomenalist materialism (Chapter 6.1). In relation to the patients' normal daily life, the ontology is the common sense one where agency (Chapter 2.1) on part of persons is simply taken for granted. The biomedical paradigm has never said that patients should be treated simply as machines, even though it was not until the second half of the twentieth century that it became an official norm that physicians have to respect the integrity of their patients (and, where this is not possible, respect the views of some close relatives or friends). In the biomedical paradigm, to repeat:

- there is no complete denial of the existence of mental phenomena

- it is taken for granted that brain states can cause mental phenomena

- there is a denial that something mental can cause a bodily medical change

- mental phenomena are regarded as being phenomena within the spatiotemporal world.

From a philosophical point of view, this list immediately raises two questions: 'What characterizes mental phenomena?' and 'What characterizes here causality?' Crucial to the contemporary philosophical characterization of the mental are two concepts: 'qualia' and 
'intentionality'. Our presentation of the causal problem will bring in the concepts of 'mental causation' and 'agency'.

\subsubsection{Qualia and intentionality}

The following kind of situation is quite common. I look at a red thing from a certain perspective and you from another; since the light is reflected a bit differently in the two directions, I see a certain hue of red but you see another. These two hues of red belong to our different perceptions as mental conscious phenomena, and they are examples of qualia. To suffer from tinnitus is to suffer from the existence of a certain kind of qualia. Whatever the head and the brain looks like, a person that does not hear anything cannot have tinnitus. There are qualia in relation to all the classical sensory systems. A person born blind cannot really know what it is like to have visual qualia; a person born deaf cannot really know what it is like to have auditory qualia. Pains are another kind of qualia. They exist only as mental apprehensions. In a mind-independent world there are no pains, only pure pain behavior. In order to find out whether or not a certain anesthesia works, one has to find out if those who receive it still feels pain or not. Corpses, and persons in coma or dreamless sleep, have no qualia. They cannot feel any pain, but, on the other hand, neither can they feel anything pleasant. A quale is a mental phenomenon. The existence of qualia is no anomaly to the biomedical paradigm.

Intentionality means 'directedness' and 'aboutness' in a sense now to be explained. When I think of past and future events, I am directed towards and think about them; when I have a desire towards something, my desire can be said to be about this something; when I am angry at somebody, my anger is directed towards and is about this person; and so on. Most mental states and acts contain intentionality, but having the feature of intentionality should not be considered a necessary condition for something to be mental. For instance, some experiences of pure qualia lack intentionality. Whether having the feature of intentionality is in general a sufficient condition for there to be a mental phenomenon, is a question that we will not consider, but, definitely, we do not today ascribe intentionality to dead matter and plants. To be a quale is in itself to be a mental phenomenon, but there can be mental states and acts even if there are no qualia; thinking, for instance, can exist unaccompanied by qualia. 
The aboutness characteristic of intentionality comes out vividly if one focuses on spatial and temporal relations. At each moment of time, dead matter and plants are completely confined within their spatiotemporal region. Not so with us. Our bodies are, but we can nonetheless think and talk of things that are far away from the spatiotemporal region that our bodies happen to occupy. Even perception is an intentional phenomenon. For instance, when we perceive another person, we are directed at something that exists at another place in space than our perceiving bodies do. Our perception is in a sense about the other person. In ordinary perceptions, intentionality and qualia are intimately fused.

Perhaps the most peculiar feature of the directedness and aboutness of intentionality is that it can be directed towards, and be about, entities that do not exist at all. We talk more or less every day about fictional figures from novels and cartoons, and these figures do neither exist in space and time nor in some other kind of man-independent realm (such as the realm of ideas postulated by Plato or the realm of mathematical numbers as postulated by many mathematicians). Nonetheless, we can identify and reidentify these figures, be it Hamlet or Superman, in such a way that a conversation about them is possible. The same goes for what is false in false assertions. If such assertions were not about anything, they could not be false. If fictional literature and cartoons were not about anything, we would not read them. False assertions and fictional assertions are similar in that none of them refers to anything in reality that corresponds to them exactly, but they differ in that false empirical assertions only as a matter of fact lack truthmakers, whereas fictional assertions cannot have any. No inorganic matter and no plants can have this kind of directedness and aboutness.

In summary, individual intentional states and acts can, although anchored in our body (especially our brain) at a certain time, be directed towards entities that:

1. are spatially distinct from the body

2. are both in the past, in the present, and in the future

3. do not in the ordinary sense exist at all. 
The third point may seem remarkable. Does it not imply the logical contradiction 'there are non-existent things'. No, it doesn't. It implies only: 'there are intentional states and acts that are directed towards non-existent things'. The fact that an intentional state or act is about something does not imply that this something can exist independently of acts of apprehension of it. Now, since in everyday language we speak as if fictional figures really exist ('Have you read the last book about Harry Potter?'), one might perhaps have better say that falsities and fictional figures exist, but that they have a special mode of existence. They can only exist in and through intentional states and acts of human beings, but they can nonetheless be the same (be re-identified) in many different such intentional acts.

Having made clear that in one sense fictions do not exist, but in another they do exist, we would like to point out that most measurement scales in medicine and the natural sciences (blood pressure, mass, electric charge, etc.) are constructed without any constraint that every magnitude on the scale has to refer to a really existing quantity in the world. Many magnitudes must be taken as having fictions as referents; it seems odd to think that all the infinitely many magnitudes of continuous scales have referents. Similarly, it makes good sense to speak of entities such as purely hypothetical kinds of genomes. In this sense there are fictions in science as well as in novels and cartoons, but this does not imply that fictions exist in some mind-independent realm of their own. Often, in both novels and science, the fictional is mixed with the real (compare the comments on 'fictionality content' in Chapter 3.5).

Intentional states and acts seem to be able to inhere in at least humans and some other higher animals, but not in pure matter and plants. What then about texts and pictures? Are they not pure matter, and mustn't they be said to contain intentionality? No, a further distinction is here needed. Texts and pictures have only a derived, not an intrinsic, form of intentionality. They can cause specific intrinsic intentional states and acts in beings with consciousness, but they do not in themselves contain the kind of directedness and aboutness that we have when are reading the texts and are seeing the pictures.

We call texts and pictures 'representations', as if they in and of themselves were directed towards and were about (represent) something distinct from themselves. But this way of speaking is probably due to the 
fact that in everyday life we take our own presence so much for granted, that a distinction between intrinsic and derived intentionality is of no pragmatic value. But in the 'ghostly' context now at hand, such a distinction is strongly needed.

It is in relation to derived intentionality, especially words and sentences, that talk of meaning and symbolic significance is truly adequate. Nouns, verbs, adjectives, and adverbs have meaning because (i) they can cause intrinsic intentional states, and we can by analytical thinking (ii) divide such signs/terms into two parts: (iia) a purely graphical sign and (iib) what contains the directedness in question. The latter is the meaning (symbolic significance), and the former, the graphical sign, has meaning (symbolic significance). The same meaning can be connected to different graphical signs (e.g., the German word 'Hund' and the English word 'dog' have the same meaning), and two different meanings can be connected to the same graphical sign (e.g., 'blade' as meaning the cutting part of things such as knives and machines and as meaning the leaf of plants).

\subsubsection{Intentional states and brain states}

Only when something with derived intentionality interacts with a brain can the corresponding intrinsic intentionality come into being. In other words, only when a representation of $\mathrm{X}$ (entity with derived intentionality) interacts with a brain can there be a real representation of $\mathrm{X}$, i.e., an intentional state or act that really is about $\mathrm{X}$. The sentence 'Clouds consist of water' is a representation of the fact that clouds consist of water only because it can cause people to be directed towards this fact and have thoughts about it.

Obviously, the feature of intentionality is not to be found among any of the scalar properties of physics and chemistry (length, mass, energy, etc.), but neither is it to be found among vector properties such as velocity, acceleration, and field strength. The directedness and aboutness of intentionality must not be confused with the directionality of motions and forces.

Intentionality is not even to be found in what quantum physicists call 'physical information' or what molecular biologists call 'genetic information'. When an intentional state contains information there is either a true assertion or a true belief about some state of affairs. But even though 
in fact true, the same kind of intentional states might have been false. Assertions and beliefs lay claim to be about something, and if this something does not obtain they are false. Assertions and beliefs have a true-falsity dimension built into their very nature. Nothing of the sorts is to be found in what is called information in the natural sciences. A distinction between two kinds of information is needed. Assertions and beliefs can contain 'intentional(-ity) information', whereas physical information and genetic information exemplify 'non-intentional(-ity) information'

According to (some interpretations of) quantum physics, there is 'physical information' that can move between 'entangled states' faster than light and 'inform' one of the entangled states about what has happened in the other. But such states contain neither intrinsic nor derived intentional directedness towards the other state; they completely lack a true-falsity dimension.

Genetic information exists in the double helix two-molecule combination DNA, and it can be represented by so-called DNA sequences consisting of a number of individual letters chosen (for human beings) out four different types of letters (A, C, G, T), each of which represents a certain nucleotide. Such information can be transferred from DNA to other molecules, in particular to 'messenger-RNA molecules', which, in turn, can transfer the information to places where the protein syntheses that create new cells can take place. In the sense of information used here, things such as vinyl records, tapes, and cd's can be said to contain information about melodies. And bar codes on commodities can be said to contain information about prizes. In all these cases, to speak about 'information' is a way of speaking about complicated causal processes where the internal structures and patterns of the causes and effects are necessary to take into account; here, the causes and effects are not simple events such as a person turning on a switch or a bacterium entering the body (as in Chapter 6.2). Biological information that resides in chemicals is not like the derived intentionality that resides in texts. The information contained in DNAs consists of patterns constituted by four different kinds of nucleotides that play a certain role in some purely non-intentional processes (taken for granted that no superhuman deity has created DNA the way humans create cd's). 
In the natural-scientific use of 'information' now made clear, i.e., 'nonintentional information', our perceptual systems can be said to receive perceptual information about the environment even when there are no associated intentional perceptual states (or acts). Perceptual psychology has made the expression 'to perceive' ambiguous. Today, it can mean both 'to receive perceptual information' and 'to be in a perceptual intentional state'. What makes the problem of perceptual intentionality even more confusing is that in order for us as persons to have veridical perceptual intentional (mental) states, our brains have to receive some corresponding perceptual information. However, this fact cannot possibly cancel the conceptual distinction between intentional and non-intentional information. Therefore, nor can it in itself show that brain states (and/or processes) containing perceptual non-intentional information are identical with the corresponding intentional states. Put in another way, the fact that brain states can (even without external stimuli) cause intentional states (even dreams are intentional states) does not show that intentional states are brain states; at least not in the way the latter are conceived of in today's physics, chemistry, and molecular biology.

When thinking about philosophical identity problems such as those concerned with brain states and intentional states, one should be acutely aware of the fact that ordinary language often relies heavily on the context and the speakers' background knowledge. For instance, to say in our culture 'Sean Connery is James Bond' is to say that Sean Connery is (playing) James Bond, not that SC and JB are identical. Similarly, nothing can be wrong in saying 'intentional states are brain states' as long as one means that intentional states are (caused by) brain states.

That there is an oddity in a complete identification of intentional states with brain states can be illustrated as follows. Touch your head with your hands. You have now a tactual percept of the outside of your head. Assume, next, that this percept is completely identical with some of your brain states. If so, what you perceive as happening on the outside of your head must in fact be happening inside your head. And the same must be true of all your veridical perceptions of events in the external world; they seem to exist outside your head, but (on the assumptions stated) they only exist inside of it. If one thinks that all intentional states are completely identical with one's brain states, then one implicitly places one's percepts 
of the ordinary world in one's brain. On such an analysis, veridical perceptions differ from dreams only in the way they are caused: dreams only have proximate causes, but veridical perceptions have distal causes too.

To accept that there are mental phenomena (qualia and intentional states) that in some way or other are connected to or inhere in the body is to accept property dualism. This dualism differs from Descartes' substance dualism in that it is not assumed that what is mental can exist apart from what is material. According to property dualism, mental phenomena can inhere in matter even though they are not like properties such as shape and volume. Property dualism is compatible with a naturalist outlook. Qualia and intentional phenomena exist in the spatiotemporal world, but they differ in their very nature from everything that so far has been postulated in physics, chemistry, and molecular biology.

\subsubsection{Psyche-to-soma causation}

Without intentional states there can by definition be no placebo effects; these effects are by definition caused by self-fulfilling mental expectations, and such expectations are intentional states. If there are neither intentional states nor qualia there are no mental phenomena at all and, consequently, no psychosomatic effects whatsoever. In order for there to be any psychosomatic effects there have to be mental phenomena, but, equally trivially, there also has to be a causal relation that goes from the mental to the bodily, from psyche to soma. In contemporary philosophy, the possibility or impossibility of such a relation is discussed under the label 'mental causation', but we will call it 'psyche-to-soma causation'. Although causal talk is ubiquitous in both everyday life and scientific life, the notion of causality is philosophically elusive (cf. Chapter 6.2). The special problem that pops up in the context now at hand is that the causes and the effects are of radically different kinds.

Conspicuous cases of causality are those where one material body affects another: a stone breaks a window, a saw cuts a piece of wood, a billiard ball pushes another billiard ball, etc. Here, some philosophical reflection may even find a metaphysical explanation: since two material bodies cannot be in the same place at the same time, something simply has to happen when two material bodies 'compete' for occupying in the same 
place. Such a kind of reasoning cannot be used when it comes to psycheto-soma causation. Mental phenomena do not compete with matter about spatial regions. What then about an electrically charged particle in an electromagnetic field? The field causes the particle to move even though they occupy the same place and they are not of exactly the same ontological kind. Instead of soma-to-soma causation there is field-to-soma causation. This is closer to psyche-to-soma causation, but the difference between the mental and the bodily seems to be much greater than that between electromagnetic fields and electrically charged particles. However, if the epiphenomenalistic materialism of the biomedical paradigm is already taken for granted, one might argue as below. The form of the argument to be presented is the indirect form that is used in RCTs: assume the opposite of what you hope to prove (namely that the null hypothesis is false), and then prove that your assumption (the null hypothesis) cannot be true.

Assume that psyche-to-soma causation is impossible. For reasons of symmetry, it then follows that even soma-to-psyche causation is impossible. Surely, this must be wrong. This means that all our experiences that being hit hard creates pain must be illusory, and that all our knowledge that alcohol and drugs can influence mental states is only presumed knowledge. The fact that somatic changes may cause mental changes is not a fact related only to the biomedical paradigm; it is a fact that is generally accepted. That bodily events can cause pain, is in common sense as obvious as the fact that one billiard ball can cause another such ball to move. Therefore, for reasons of symmetry psyche-to-soma causation is just as possible as soma-to-psyche causation, which, in turn, according to everyday perception, is just as possible as soma-to-soma causation.

\subsubsection{Agency}

So far, we have spoken of psyche-to-soma causation the way we spoke of causal relations between purely material events, soma-to-soma causation. Even agency (Chapter 2.1) is, if it exists, a kind of psyche-to-soma causation; one which brings in free will and human freedom. It shall explain why soft (and not hard) determinism is true. This issue, let it be noted, is of no relevance for the question of the existence of placebo and nocebo effects and other passive psychosomatic processes. But since it 
belongs to the general issue of psyche-to-soma causation, and is a necessary condition for the existence of what we termed active psychosomatic curing, we will take the opportunity to make some remarks on it here; especially, since experiments by a physiologist, Benjamin Libet (b. 1916), has become prominent in the discussion.

Agency contains a special kind of intentionality: intentions. Obviously, some unconscious electric processes in the brain are necessary preconditions for our actions; without a brain no agency. In Libet's experiments, the actions were very simple ones such as pressing a button, which we know are correlated with neuron activity in the supplementary cortex. If his results are generalized to all kinds of actions, one can state his conclusions as follows. The neurons in the brain that are responsible for a certain physical movement in a certain body part start firing about 500 milliseconds before this movement takes place, but conscious intentions or urges to make such a movement/action arise about 150 ms before the action starts. That is, seemingly free actions are triggered about (500$150=) 350$ ms before the presumably free urge or free decision to act appears. It might be tempting to conclude that the experienced decision to act is merely an epiphenomenon to the first $350 \mathrm{~ms}$ of the relevant brain processes, and that we should look upon agency as a complete illusion. Libet's own conclusion, however, is not that radical. He says that we are still free to inhibit actions that are on their way; there is at least $150 \mathrm{~ms}$ left for intervention by a free will. On his view, true agency can only be controlling, stopping some actions and letting others through.

As we have said, science and philosophy overlap. One kind of criticism leveled at Libet from a neurologist-philosopher pair says that he has neglected the fact that voluntary actions need not be preceded by any felt urge or decision to act (Bennet and Hacker, 8.2). Let us put it as follows: sometimes we have in the mind's eye a specific intention to act before we act, but often we become aware of our intention only in our very acting. That is, there are two kinds of intentions, reflective (or prior) intentions and non-reflective intentions. Libet seems to think that all free actions require reflective intentions.

The distinction between reflective and non-reflective intentions is quite in conformance with common sense and judicial praxis. We talk of children as performing actions long before they are able to have any 
reflective intentions; they are said to act spontaneously, not to be mere stimulus-response machines. We even take it for granted that we often directly in a movement can see whether or not it is an action or 'mere movement'; and we cannot see reflective intentions, only be told about them. For instance, in soccer, where playing with the hands is forbidden, the referees are expected to be able to see whether or not an arm that touches the ball is intentionally touching it. If there is an intention, they give a free-kick; otherwise not. Usually, players have no time to form prior intentions before they are acting. In most laws, ever since ancient times, there is some distinction between law-breakings that are done reflectively (e.g., murder) and the same ones done un-reflectively (manslaughter). In judicial Latin, they are called 'dolus' and 'culpa', respectively.

\subsection{Biomedical anomalies}

A scientific anomaly is some empirical datum or unified collection of empirical data that cannot - at a certain time - be accommodated by the current paradigm. The future will tell whether an anomaly is in fact reality's way of falsifying the paradigm, or if it is merely a symptom of a need to develop the paradigm further. Anomalies should be kept conceptually distinct from paradigm conflicts, even though such conflicts can contain mutual accusations that the other paradigm is confronted with falsifying anomalies. In this vein we have already discussed alternative medicine. Before discussing what might be regarded as present-day anomalies to the biomedical paradigm, we will illustrate the concept of anomaly a little more than we have done so far (see Chapter 2.4). History of astronomy provides an illustrative example of how similar kinds of anomalies can be either falsifications or merely cries for improvement. The same two possibilities exist in relation to biomedical anomalies too.

During ancient and medieval times, scientists claimed that not only the Moon, but also the Sun, the planets and the stars move around the Earth in certain orbits (the geocentric worldview). Since the Renaissance, we believe the opposite: the planets, now including the Earth, orbit around the Sun (the heliocentric worldview). Today, we also know that our solar system moves in relation to other solar systems. After the heliocentric theory had become accepted, Isaac Newton proposed his famous mechanics. This theory is meant to explain movements of all kinds of 
material bodies. We shall highlight some of its anomalies in relation to our solar system.

Only the orbits of a few planets were in precise accordance with the calculations based on Newtonian mechanics. Especially significant were the deviations for Mercury and Uranus (the latter planet discovered after Newton's death). Newton's law of gravitation says that the force between two interacting bodies is determined by the masses of the bodies and the distance between them. This does not mean, however, that a planet's orbit around the Sun is only dependent on the mass of the planet, the mass of the Sun, and the distance between these bodies. The planets also influence each other mutually. Therefore, scientists could easily attempt to rescue Newton's theory from the anomaly of Uranus's orbit by proposing an auxiliary hypothesis (see Chapter 4.4.), namely that there is another planet - not yet discovered - that also influences the movement of Uranus. Scientists calculated what the mass and the orbit of the hypothetical planet would have to look like if the planet should be able to explain Uranus' actual deviation from the earlier calculated orbit. After having calculated also an actual position of the still hypothetical planet, they directed their telescopes towards this place in the heavenly sphere - and, what a wonder - they discovered the planet; it was baptized 'Neptune'. However, when the actual orbit of Neptune was plotted, one discovered that it did not conform to the calculated one. That is, the solution to one anomaly gave rise to another anomaly. What to do? Well, the strategy was clear. One started to search for another planet and found Pluto. This is a wonderful research story. (Let it be noted, that in August 2006 it was decided that Pluto should no longer be classified as a 'planet' but as a 'dwarf planet'; some words about this in Chapter 8.2.)

What then about the anomaly caused by the funny orbit (a rotating ellipse) of Mercury? Even in this case the astronomers tried to explain the anomaly by means of an auxiliary hypothesis that posited an unknown planet. This planet was assumed to be situated between Mercury and the Sun, and it was given the name 'Vulcan'. One searched and searched for Vulcan, but it could never be found. Later, the orbit of Mercury became explained by the theory of general relativity. In retrospect we can say that the auxiliary hypothesis failed to explain the anomaly. 
Let us now return to the biomedical paradigm and its anomalies. We can now ask if contemporary medical researchers should only search for 'unknown planets' or for new kinds of 'gravitational fields' too. Should they look only for new physiological, biochemical, and microbiological mechanisms or for something else as well? Psychosomatic diseases and the placebo effect are anomalies that bring in psyche-to-soma causation, but there are other anomalies that do not necessarily involve this problem. We will comment on the phenomenon of psychosomatic diseases in the next section but on the placebo effect at once.

The placebo effect is an anomaly in the biomedical paradigm, but it is nonetheless a normal part of the clinical medical paradigm since it is an explicit part of many RCTs. How come? Shouldn't the clinical medical paradigm be considered a sub-paradigm to the biomedical paradigm? The explanation is that the placebo effect is in RCTs used only as a means to find what is regarded as a biomedical effect, which is based in a soma-tosoma causality. There is so to speak only a negative acceptance of the placebo effect, and this, we guess, is the reason why the placebo effect only creates a bit of a tension, a cognitive dissonance, between the clinical medical paradigm and the biomedical paradigm. But this is an unhappy state of affairs. The placebo effect should be considered an anomaly and be treated as such. That is, one should either try to show that there simply is no placebo effect or try, positively, to investigate the placebo effect as such. In the former case, the anomaly is explained away because the biomedical paradigm becomes more developed in relation to regression fallacies and studies of the natural courses of diseases and illnesses; in the latter case, today it is impossible to say what would happen to the biomedical paradigm if its soma-to-soma and soma-to-psyche causal relations would have to be truly complemented with psyche-to-soma relations.

But there is also another kind of anomalies, well known to e.g., physicians. Concepts such as 'specific symptoms', 'disease', 'etiology', 'pathogenesis', and 'specific treatment' belong to the normal everyday discourse of clinicians and are central to the biomedical paradigm. To claim that a symptom is specific is to claim that it is the sign of a specific underlying pathological anatomical state/structure or a pathological physiological process. Prototypical examples are: dysfunction of the 
endocrine system, deficiency diseases, chromosome aberrations, genetic diseases, infectious diseases, and cancer diseases. As previously stated, a treatment is specific when we have knowledge about the underlying pathological mechanism and where and how the treatment interferes in the mechanism.

The ideal of the biomedical paradigm is to discover and describe specific symptoms and diseases, and on such a basis develop specific treatments. By means of symptoms physicians should be able to diagnose an underlying disease and, knowing the disease, they should know what treatment to give. However, this ideal is at present, at least for GPs, impossible to attain. Several symptoms cannot be connected to any known underlying pathological structure or mechanism, and many symptoms simply fade away without leaving any clues as to what the patient was specifically suffering from. Sometimes the underlying disease is not wholly understood or only partly known, and then GPs speak of 'symptoms diagnoses'; and sometimes the symptoms constitute such a complex pattern that they speak of 'syndrome diagnoses'.

Physicians have given some of these unspecific symptoms and diagnoses nicknames such as 'address-less symptoms', 'diagnoses of exclusion', and even 'kitchen sink diagnoses'. Unspecific symptoms of the back, the chest, the head, and the urethra are examples of address-less symptoms, and the Irritable Bowl Syndrome (IBS) is an example of a diagnosis of exclusion. Often, unspecific symptoms are also called 'functional inconveniences', and the patients suffering from them are sometimes described as tricky, difficult, heavy, hysterical, or hypochondriac; sometimes even as malingerers.

One might wonder why physicians so readily speak disparagingly about patients for whom they are not able to make a specific diagnosis. Arguably, there is a general tendency in human nature to blame the things one cannot cope with rather than to blame one's own capacity or the knowledge of the community to which one belongs. But perhaps a better understanding of the philosophy-of-science phenomena of anomalies can alter this behavior. If anomalies are normal, then neither the doctors nor the patients need to be blamed when problematic symptoms and disease patterns occur. The moral of the story is that some major discoveries are made when researchers concentrate on anomalies. 


\subsection{Helicobacter pylori in the machine}

According to the views of science and philosophy that we have presented, there is no simple litmus test that can tell what specific theory is the most truthlike, or what paradigm one should work with in the future. The positivist dream is gone. But, we also said, paradigms and theories have to be evaluated; anything does not go. Reality constrains our conceptual schemas. To regard the human body as a social construction is psychologically possible only for people who do not suffer from any serious somatic disease. Fallibilist epistemological and ontological realism is the golden mean between positivism and social constructivism. Let us now take a look at the case of peptic ulcer and Helicobacter pylori in the light of what has been said about correlations, black boxes, grey boxes, and shining mechanisms.

Peptic ulcer was clearly identified in the nineteenth century thanks to the increased use of autopsies. It was first discovered in the stomach and later on in the duodenum. Up until the 1930s, the cause of it was regarded to be purely biomedical; hyperacidity was regarded as the proximate cause and (in some corners) spicy food as the distal cause. At that time surgery was a common treatment, cutting off nerves which were supposed to stimulate the production of acids in the stomach. But then one started to question if such factors as personality traits, psychological trauma, and psychological stress could cause peptic ulcer. That there is a correlation between psychological stress and peptic ulcer seemed obvious to many people from mere everyday observations. And the biomedical paradigm didn't have a grey box yet, i.e., an outline of some mechanism. At this time, it was more or less a scientific fact that bacteria cannot live in strongly acidic environments in the stomach. In many scientific disciplines such as social medicine and stress research, it was during the second half of the twentieth century a commonplace that peptic ulcer is wholly a psychosomatic disease. This view also influenced textbooks and medical education programs.

In the early 1980s, two Australians, the pathologist Robin Warren (b. 1937) and the physician Barry Marshall (b. 1951), published a couple of papers claiming that there is a spiral-shaped bacterium (now named Helicobacter pylori) that causes peptic ulcer and gastritis. That is, there is a 


\section{Helicobacter pylori}

- the bacterium causing peptic ulcer disease
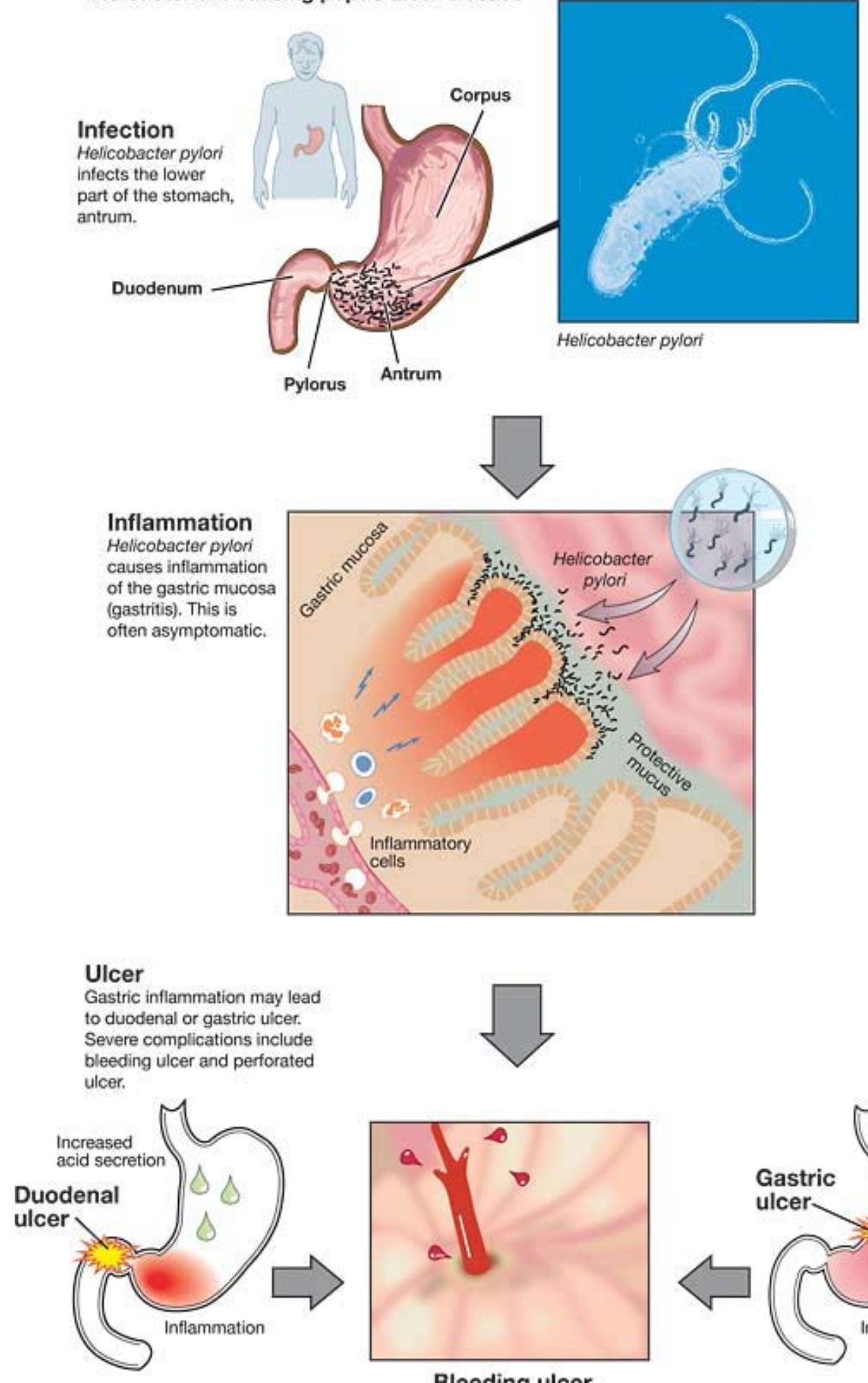

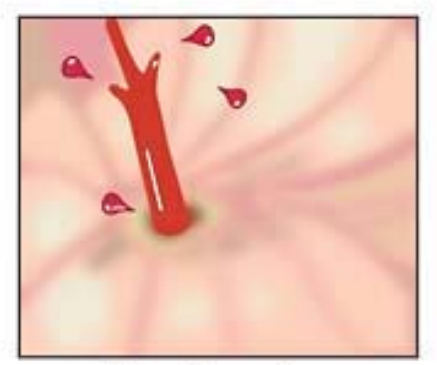

Bleeding ulcer

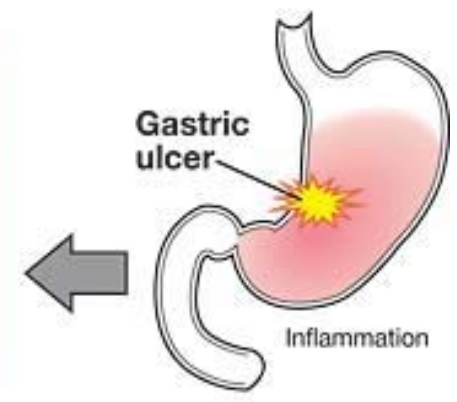

() The Nobel Committee for Physiology or Medicine

Figure 3: From the press release of the 2005 Nobel Prize in Physiology or Medicine (from the Nobel Assembly at Karolinska Institutet). 
bacterium that can thrive in acidic milieus. In fact, this bacterium had been observed by German scientists in the nineteenth century, but these observations were forgotten since no one was able to isolate it and grow it in a culture (compare Koch's second postulate; Chapter 2.4). Warren and Marshall, however, could isolate the bacterium and show that it was present in most cases of peptic ulcer and gastritis (cf. Koch's first postulate). Marshall belongs to the list of courageous medical researchers who made tests on themselves. He drank a Petri dish of $\mathrm{H}$. pylori and developed gastritis (cf. Koch's third postulate), whereupon the bacteria could be found in and re-isolated from his stomach lining (cf. Koch's fourth postulate). Also, they showed that $\mathrm{H}$. pylori can be eradicated by means of antibiotics. Patients with chronic peptic ulcer that previously had to suffer from the disease more or less chronically can now recover completely. Later, beginning in the mid 1990s, the official government health authorities of many countries turned Warren and Marshall's views into an official doctrine. In 2005, W\&M received the Nobel Prize in medicine. Before their discovery, peptic ulcer and gastritis were mostly treated with medicines that neutralized stomach acid or decreased its production.

What now to say about the H. pylori discovery from a philosophicoscientific point of view? First, it refutes all those who thought that no microbiological mechanism that explains peptic ulcer could ever be found. Second, because of the problem of induction (Chapter 4.2), the discovery cannot without further ado be taken as having shown that all talk about psychosomatic diseases must be illusory talk. Third, and more interestingly, it does not completely eliminate the peptic ulcer anomaly. Something more has to be said. It is estimated that:

(a) as much as $85-90 \%$ of all persons that are infected by $\mathrm{H}$. pylori are asymptomatic and do not develop any ulcer (50\% of all humans have this microbe), and

(b) H. pylori is not a causal factor in all ulcer cases; it accounts for $75 \%$ of gastric ulcer and $90 \%$ of duodenal ulcer.

Let us now make use of the concept of 'component cause' ('INUScondition') that we presented in Chapter 6.2. Points (a) and (b) imply that 
H. pylori is a component cause of peptic ulcer. It is not in itself sufficient to produce peptic ulcer, but it is a necessary part of some kinds of causal mechanisms that are sufficient to produce peptic ulcer; however, peptic ulcer can also be produced by mechanisms that do not contain H. pylori at all. The abstract structure of the causal problem can now be illustrated as follows.

Assume that there are three kinds of mechanisms $\left(\mathrm{M}_{1}, \mathrm{M}_{2}, \mathrm{M}_{3}\right)$ that need to have $\mathrm{H}$. pylori $(\mathrm{H})$ as a part in order to produce gastric ulcer, and that there is only one kind $\left(\mathrm{M}_{4}\right)$ that does not need this bacterium. Furthermore, assume that all these mechanisms can be divided into three main parts (A, $\mathrm{B}, \mathrm{C}$, etc). That is, there are four different situations:

1. $\mathbf{M}_{1}(=\mathrm{A} \& \mathrm{~B} \& \mathbf{H})$ produces gastric ulcer

2. $\mathbf{M}_{2}(=\mathrm{C} \& \mathrm{D} \& \mathbf{H})$ produces gastric ulcer

3. $\mathbf{M}_{3}(=\mathrm{E} \& \mathrm{~F} \& \mathbf{H})$ produces gastric ulcer

4. $\mathrm{M}_{4}(=\mathrm{J} \& \mathrm{~K} \& \mathrm{~L})$ produces gastric ulcer

According to these assumptions, $\mathrm{H}$. pylori is a cause of gastric ulcer in three cases out of four (75\%). In relation to the fourth case, we can still ask the traditional question whether anything purely mental can constitute the whole of $J \& K \& L$ and be sufficient to produce an ulcer; psychological stress need only be one of the parts in J\&K\&L. In relation to the first three cases, we can ask if any of the cofactors to $\mathrm{H}$. pylori, A to F, can be identical with psychological stress. If the answer is 'yes', there is still a problem of how to understand a psyche-to-soma relation, but this time it needs perhaps not be a strictly causal relation.

Assume that ' $E$ ' represents psychological stress; and that $E$, the physiological conditions $\mathrm{F}$ and $\mathrm{H}$. pylori together produce gastric ulcer. We do not then necessarily have to interpret the \&-signs in ' $\mathrm{E} \& \mathrm{~F} \& \mathrm{H}$ ' as representing casual interaction. Our assumptions only force us to say that there is a (psyche+soma)-to-soma causality, i.e., (E\&F\&H)-to-ulcer causality, not that there is a pure psyche-to-soma causality. However, instead we arrive at another problem: how can something mental and something somatic be non-causally connected? How can a bacterium $(\mathrm{H})$, a physiological condition (F), and a psychological state (E) make up a unity that can function as a cause? 
Let us leave our speculations at that. As we have already said once in this chapter, we do not consider the problems of the existence of psychosomatic diseases and placebo effects to be solved. This book is meant to introduce the readers to such problems and to ways one might think and speculate about them, not to tell the readers what the true answers are. Science and philosophy will never come to an end. We will conclude this chapter with historical remarks.

In our opinion, the problem of the psyche-to-soma and the soma-topsyche causal relations look neither worse nor better than some causality problems modern physics faces. For instance, Newtonian mechanics has a problem with action-at-a-distance. According to many intuitions both inside and outside philosophy, things that cause and things that are caused have to be in contact with each other, i.e., causality implies contact. But this is not the case in Newton's theory. (The theory of special relativity does not, by the way, solve this problem; rather the contrary, since it says that no form of energy can move faster than light.) According to the law of gravitation, any two material particles in the whole universe, whatever the distance between them, affect each other instantly. Think of the Sun and the Earth. How can they affect each other instantly when they are eight light minutes away from each other? The problem was clearly perceived by Newton himself, and he simply declared the law to be a 'phenomenological law'; and he explicitly said that he did not make any hypotheses about the causal mechanism.

Field theories lack this problem; in such theories interacting particles have their interaction mediated by a field that connects the particles. One electrically charged particle can influence another if there is an electric field emanating from the first particle that affects the other. In the direct field-to-particle interaction there is then action by contact. However, there still seems to be no really good theory available for gravitational fields.

In modern quantum mechanics, the problem of action-at-a-distance reappears in a completely different kind of mathematical formalism and under a new name: 'entanglement'. Two entangled particles (at any spatial distance from each other) are assumed to be able to exchange physical information instantly (but not to transmit energy signals faster than light). The equations allow physicists to make predictions, and so did Newton's laws, but a causal mechanism seems wanting. The problems of action-at-a- 
distance and entanglement can be said to be problems of how to conceive what is spatially disconnected as being nonetheless connected. The problems of psyche-to-soma and soma-to-psyche relations can be said to be problems of how to conceive that entities that seemingly have radically distinct ontological natures can nonetheless have something in common.

\section{Reference list}

Album D. Sykdommers og Medisinske Spesialiteters Prestisje [The prestige of illnesses and medical specialities]. Nordisk Medicin 1991; 106: 232-6.

Balint M. The Doctor, his Patient and the Illness. Churchill Livingstone. London 1964.

Bloch M. The Ryoal Touch: Monarchy and Miracles in France and England. Dorset Press. New York 1961.

Bootzin RR, Caspi O. Explanatory Mechanism for Placebo Effects: Cognition, Personality and Social Learning. In Guess HA, Kleinman A, Kusek JW, Engel LW (eds.). The Science of the Placebo. Toward an Interdisciplinary Research Agenda. BMJ Books. London 2002.

Cabot RC. The Physician's Responsibilty for the Nostrum Evil. Journal of the American Medical Association 1906; 57: 982-3.

Cannon WB. Voodoo Death. American Anthropologist 1942; 44: 169-81.

Cobb L, Thomas GI, Dillard DH, et al. An evaluation of internal-mammary artery ligation by a double blind technic. New England Journal of Medicine 1959; 260: 1115-8.

Cohen S. Voodoo Death, the Stress Response, and AIDS. In Bridge TP, Mirsky AF, Goodwin FK (eds.). Psychological, Neuropsychiatric, and Substance Abuse Aspects of AIDS. Raven Press. New York 1988.

Davies CE. Regression to the Mean or Placebo Effect? In Guess HA, Kleinman A, Kusek JW, Engel LW (eds.). The Science of the Placebo. Toward an Interdisciplinary Research Agenda. BMJ Books. London 2002.

Dorland Medical Dictionary. 30th edition 2003.

Eisenberger NI, Lieberman MD, Williams KD. Does Rejection Hurt? A fMRI Study of Social Exclusion. Science 2003; 302: 290-2.

Guess HA, Kleinman A, Kusek JW, Engel LW (eds.). The Science of the Placebo. Toward an Interdisciplinary Research Agenda. BMJ Books. London 2002.

Harrington A. Seeing the Placebo Effect: Historical Legacies and Present Opportunities. In Guess HA, Kleinman A, Kusek JW, Engel LW (eds.). The Science of the Placebo. Toward an Interdisciplinary Research Agenda. BMJ Books. London 2002. 
Hrobjartsson A, Gotzsche P. Is the Placebo Powerless? An Analysis of Clinical Trials Comparing Placebo with No Treatment. New England Journal of Medicine 2001; 344: 1594-1602.

Jarvinen KA. Can ward rounds be a danger to patients with myocardial infarction? British Medical Journal 1955; 4909: 318-20.

Kleinman A, Guess HA, Wilentz JS. An overview. In Guess HA, Kleinman A, Kusek JW, Engel LW (eds.). The Science of the Placebo. Toward an Interdisciplinary Research Agenda. BMJ Books. London 2002.

Leibovici L. Effects of remote, retroactive intercessory prayer on outcomes in patients with bloodstream infection: randomised controlled trial. British Medical Journal 2001; 323: 1450-1.

Lieberman MD, Jarcho JM, Berman S, et al. The Neural Correlates of Placebo Effect: a Disruption Account. NeuroImage 2004; 22: 447-55.

Moerman, DE. Meaning, Medicine and the "Placebo Effect". Cambridge University Press 2002.

Moerman DE. Explanatory mechanism for placebo effects: cultural influences and the meaning response. In Guess HA, Kleinman A, Kusek JW, Engel LW (eds.). The Science of the Placebo. Toward an Interdisciplinary Research Agenda. BMJ Books. London 2002.

Moseley JB, O’Malley K, Petersen NJ, et al. A Controlled Trial of Arthroscopic Surgery for Osteoarthritis of the Knee. New England Journal of Medicine 2002; 347: 81-8.

Olsson B, Olsson B, Tibblin G. Effect of Patients' Expectations on Recovery from Acute Tonsillitis. Family Practice 1989; 6: 188-92.

Petrovic P, Kalso E, Petersson KM, Ingvar M. Placebo and opoid anagesia - imaging a shared neuronal network. Science 2002; 295: 1737-40.

Petrovic P, Dietrich T, Fransson P, Andersson J, Carlsson K, Ingvar M. Placebo in emotional processing - induced expectations of anxiety relief activate a generalized modulatory network. Neuron 2005; 46: 957-69.

Ryle G. The Concept of Mind. Penguin Books. Harmondsworth. Middlesex 1966.

Searle J. Intentionality. Cambridge University Press. Cambridge 1983.

Silverman WA. Human Experimentation. A Guided Step into the Unknown. Oxford Medical Publications. Oxford 1985.

Thomsen J, Bretlau P, Tos M, et al. Placebo effect in surgery fot Meniere's disease: three years follow up. Archives of Otolaryngology—Head \& Neck Surgery 1983; 91: 183-6.

White L, Tursky B, Schwartz GE (eds.). Placebo - Theory, Research and Mechanism. Guildford Press. New York 1985.

Zubieta JK, Bueller JA, Jackson LR, Scott DJ, Xu Y, Koeppe RA, Stohler CS. Placebo effects mediated by endogenous opioid neurotransmission and $\mu$-opioid receptors. Journal of Neuroscience 2005; 25: 7754-7762. 